\title{
Effect of Fluorine Incorporation on the Thermal Stability of PtSi/Si Structure
}

\author{
Bing-Yue Tsui, Student Member, IEEE, Jiunn-Yann Tsai, Student Member, IEEE, \\ Tzong-Shien Wu, and Mao-Chieh Chen
}

\begin{abstract}
The effect of fluorine incorporation on $\mathrm{PtSi} / \mathrm{Si}$ structure is studied systematically. It is observed that the fluorine incorporation from ion implantation improves the hightemperature stability of the PtSi/Si structure. The optimum implantation energy is determined to be the energy at which the maximum percentage of the as-implanted fluorine ion locates at the $\mathrm{PtSi} / \mathrm{Si}$ interface region. The SIMS analysis shows that the fluorine atom piles up at the PtSi/Si interface. The XPS analysis indicates that the fluorine atoms at the PtSi $/ \mathrm{Si}$ interface are bonded to the silicon atoms in a form of $\mathrm{SiF}_{2}$ or $\mathrm{SiF}_{3}$. A fluorine-buffer (FB) model is proposed to explain the effect of fluorine incorporation. It is postulated that the Si-F layer acts as a buffer layer to change the $\mathrm{PtSi} / \mathrm{Si}$ interface energy and preserve the integrity of the silicide layer at high temperature. Fluorinated Schottky junctions are fabricated and the electrical characteristics show that the sustainable process temperature can be improved from $650^{\circ} \mathrm{C}$ for the unfluorinated Schottky junctions to higher than $800^{\circ} \mathrm{C}$ for the fluorinated Schottky junctions.
\end{abstract}

\section{INTRODUCTION}

$\mathrm{P}$ LATINUM SILICIDE (PtSi) has been widely used for its stable features as well as low resistivity. With the progress of VLSI technology, thin-film silicides become increasingly important. Formation of low-resistance contact, low sheet-resistance diffused region, and polycide gate and interconnection line is crucial for the VLSI circuit performance. PtSi can form stable ohmic and Schottky contacts to silicon at temperatures lower than $600^{\circ} \mathrm{C}$. It consumes less silicon than the refractory metals during silicide formation [1]. It is suitable for SALICIDE process [2]. It has also been reported that using $\mathrm{Pt}$ or $\mathrm{PtSi}$ as a barrier film, high-quality shallow junctions with junction depths of less than $100 \mathrm{~nm}$ (both $n^{+}-p$ and $p^{+}-n$ junctions) can be fabricated using furnace annealing at tem-

Manuscript received April 14, 1991; revised July 25, 1992. This work was supported by the National Science Council, ROC, under Contract NSC80-0404-E000-14. The review of this paper was arranged by Associate Editor K. Shenai.

B. Y. Tsui and M. C. Chen are with the Department of Electronics Engineering, and the Institute of Electronics, National Chiao-Tung University, Hsinchu, Taiwan, ROC.

J. Y. Tsai was with the Department of Electronics Engineering, and the Institute of Electronics, National Chiao-Tung University, Hsinchu, Taiwan, ROC. He is now with the Department of Electrical Engineering, North Carolina State University, Raleigh, NC 27607.

T. S. Wu was with the Department of Electronics Engineering, and the Institute of Electronics, National Chiao-Tung University, Hsinchu, Taiwan, ROC. He is now with the United Micro-electronics Corporation, Hsinchu, Taiwan, ROC.

IEEE Log Number 9204306. peratures lower than $750^{\circ} \mathrm{C}$ [3], [4]. All of these indicate that PtSi should be a choice for application to very large scale bipolar and MOS integrated circuits. However, hightemperature stability of PtSi has always been questionable.

The often quoted high-temperature instability of PtSi on silicon apparently arises from the fact that the lowest eutectic point in the $\mathrm{Pt}-\mathrm{Si}$ system occurs at $830^{\circ} \mathrm{C}$ [5]. Even after the complete consumption of metal to form silicide at low temperature, platinum diffusion into silicon substrate from PtSi at a temperature of $700^{\circ} \mathrm{C}$ or higher has been reported [6]. The high-temperature instability of PtSi has also been observed in the degradation of the $\mathrm{PtSi} / \mathrm{n}$-Si Schottky junction. Annealing of such a junction to a temperature of $650^{\circ} \mathrm{C}$ or higher will cause large reverse leakage current and nonideal forward currentvoltage characteristics [7]. One effort to improve the hightemperature instability associated with the PtSi/Si structure is to produce a film with $\mathrm{Si} / \mathrm{Pt}$ atomic ratio higher than that in the eutectic to bypass the eutectic composition and hence the instability [8]. It is shown that the co-sputtered silicon-rich alloy prevents agglomeration of the silicide, but increases the resistivity and decreases the Schottky-barrier height of the film. Electrical properties such as forward and reverse current-voltage characteristics still degrade after high-temperature annealing. Furthermore, the technique is not compatible with the modern VLSI process.

Recently, the effect of fluorine incorporation on the MOS device has become an attractive research field. Sequential experiments on the dielectric properties, the radiation hardness, and the hot-carrier immunity of the fluorinated MOS device has been pursued by Yale, Stanford, and Hitachi groups [9]-[16]. With fluorine incorporation at appropriate conditions, the interface state density and the interface radiation hardness of the $\mathrm{SiO}_{2} / \mathrm{Si}$ interface and the hot-carrier resistance of the MOSFET device are improved significantly. One drawback is that a higher dose of fluorine causes degradation in charge to breakdown [10]. Other effects associated with fluorine incorporation on MOS devices are studied by other groups. It is observed that fluorine plays a major role in the penetration of boron into and through the gate oxides of p-channel MOSFET's which employ $\mathrm{p}^{+}$-doped polysilicon gates [17]-[20]. It has also been suggested that fluorine atoms may be trapped by group $\mathrm{V}$ dopants in $\mathrm{n}$-type 
TABLE I

EXPerimental Conditions USEd to Determine the Optimum IMPlantation Condition for the High-Temperature Stability IMProvement of THE PtSi/Si Structure By BF ${ }_{2}^{+}$-ION IMPLANTATION

\begin{tabular}{lcccccc}
\hline \multicolumn{1}{c}{ Sample Structure } & \multicolumn{5}{c}{$\mathrm{PtSi}(300 \AA) / \mathrm{Si}$} \\
\hline $\mathrm{BF}_{2}^{+}$energy $(\mathrm{keV})$ & 38 & 75 & 100 & 125 & \\
$\mathrm{~F}^{+}$effective energy $(\mathrm{keV})$ & 15 & 30 & 40 & 50 & \\
$\mathrm{BF}_{2}^{+}$dose $\left(1 / \mathrm{cm}^{2}\right)$ & $5 \times 10^{13}$ & $2 \times 10^{14}$ & $5 \times 10^{14}$ & $1 \times 10^{15}$ & $2 \times 10^{15}$ & \\
Annealing temperature $\left({ }^{\circ} \mathrm{C}\right)$ & 400 & 700 & 750 & 800 & 830 & 850 \\
Annealing time & & \multicolumn{5}{c}{$60 \mathrm{~min}$} \\
\hline
\end{tabular}

silicon due to high electronegativity of fluorine atoms. Fluorine atoms prevent the $\mathrm{As}^{+}-\mathrm{Si}^{-}$and $\mathrm{P}^{+}-\mathrm{Si}^{-}$pairs forming, the formation and dissociation of which cause diffusion enhancement and extended defects. However, the formation of $\mathrm{As}^{+}-\mathrm{F}^{-}$and $\mathrm{P}^{+}-\mathrm{F}^{-}$pairs reduces the dopant activation [21].

In the authors' previous work, it was observed that implanting fluorine-contained ions (such as $\mathrm{F}^{+}$or $\mathrm{BF}_{2}^{+}$) through the $\mathrm{Pt} / \mathrm{Si}$ (Implantation Through Metal, ITM) or PtSi / Si (Implantation Through Silicide, ITS) structure allows the $\mathrm{PtSi} / \mathrm{Si}$ structure to withstand a higher process temperature, up to $800^{\circ} \mathrm{C}$, without degradation [22]. This high-temperature stability was confirmed by sheet resistance measurement, scanning electron microscopic (SEM) inspection, and Auger electron spectroscopy (AES) and Rutherford backscattering spectroscopy (RBS) analyses. Secondary ion mass spectroscopy (SIMS) analysis indicates that the implanted fluorine atoms pile up at the $\mathrm{PtSi} / \mathrm{Si}$ interface. The high-temperature stability should be correlated to the fluorine pileup. In this work, the effect of fluorine incorporation on the $\mathrm{PtSi} / \mathrm{Si}$ structure is studied systematically. The optimum condition of fluorine implantation is determined. A fluorine-buffer (FB) model is proposed to explain the improvement of high-temperature stability. Fluorinated Schottky junctions were fabricated and compared to unfluorinated Schottky junctions.

Both ITM and ITS samples were processed. Because the results of the ITS scheme are similar to those of the ITM scheme, only the ITM scheme is reported and discussed in detail. Nonetheless, some results different for the ITS and ITM schemes will be stated in the text.

\section{EXPERIMENTS AND RESUlts}

\section{A. $B F_{2}^{+}$Implantation}

In [22], the authors have clarified the effect of ion implantation on the high-temperature stability of PtSi film by implanting different species of $\mathrm{BF}_{2}^{+}, \mathrm{F}^{+}$, and $\mathrm{B}^{+}$separately at equivalent energies and doses into the $\mathrm{PtSi} / \mathrm{Si}$ samples. It was concluded that the improvement of the high-temperature stability is attributed to the fluorine ions with the boron ions playing no role. Similar results could be obtained by implanting $\mathrm{F}^{+}$or $\mathrm{BF}_{2}^{+}$ions if the fluorine ions had the same effective energy. Because the beam current of $\mathrm{BF}_{2}^{+}$ions is at least one order of magnitude higher than that of the $\mathrm{F}^{+}$ions when $\mathrm{BF}_{3}$ gas is used as the ion source, $\mathrm{BF}_{2}^{+}$were employed to introduce fluorine at first to determine the optimum implantation condition.

The starting material was n-type $(100)$ oriented $0.8-$ $2.7-\Omega \cdot \mathrm{cm}$ phosphorus-doped silicon wafer. The wafers were first cleaned by the standard RCA cleaning process. Platinum film of about $300 \AA$ thickness was deposited in an electron-beam evaporation system with a base pressure of less than $5 \times 10^{-6}$ torr and a deposition rate of $1 \AA / \mathrm{s}$. Before loading into the deposition chamber, wafers were immersed in dilute $\mathrm{HF}$ solution ( $\mathrm{HF}: \mathrm{H}_{2} \mathrm{O}=1: 50$ ) for 1 to $2 \mathrm{~min}$ to remove the surface oxide grown during cleaning, and the silicon surface was passivated by hydrogen and/or fluorine at the same time which can prohibit oxidation in air [23]. After $\mathrm{Pt}$ deposition, $\mathrm{BF}_{2}^{+}$ions were implanted into the wafers at different energies from 38 to $125 \mathrm{keV}$ to different doses from $1 \times 10^{14}$ to $5 \times 10^{15}$ $\mathrm{cm}^{-2}$. The corresponding fluorine energy was determined by multiplying the $\mathrm{BF}_{2}^{+}$energy with the ion mass ratio of $\mathrm{F}^{+} / \mathrm{BF}_{2}^{+}=19 / 49$ to be from 15 to $50 \mathrm{keV}$. Wafers were first annealed in $\mathrm{N}_{2}$ ambient at $400^{\circ} \mathrm{C}$ for $60 \mathrm{~min}$ to form $\mathrm{PtSi}$ and the unreacted $\mathrm{Pt}$ was selectively etched by boiling in dilute aqua regia. Wafers were then cut into six pieces and five of them were further annealed in $\mathrm{N}_{2}$ ambient for $60 \mathrm{~min}$ at $700,750,800,830$, and $850^{\circ} \mathrm{C}$, respectively. Table I summarizes the key experimental conditions.

The sheet resistance $\left(R_{s}\right)$ of each sample was measured by a four-point probe. For each sample before degradation, the deviation of $R_{s}$ over five measurements is within 1-2 $\Omega / \square$. Figs. 1 and 2 show the $R_{s}$-annealing temperature relation of the samples implanted with $\mathrm{BF}_{2}^{+}$ion at 38 and $75 \mathrm{keV}$ (effective $\mathrm{F}^{+}$energy of 15 and $30 \mathrm{keV}$ ), respectively; the $R_{s}$-temperature data of the unimplanted samples are also included for comparison. For the unimplanted samples, the $R_{s}$ value starts to increase at $750^{\circ} \mathrm{C}$. For the samples implanted with $\mathrm{BF}_{2}^{+}$ion at $38 \mathrm{keV}$, the $R_{s}$ value remains stable up to $800^{\circ} \mathrm{C}$ but increases drastically at higher temperatures independent of the implanted dose. For the samples implanted with $\mathrm{BF}_{2}^{+}$ion at $75 \mathrm{keV}$, a different tendency was observed. For the two lower dose sample sets, $R_{s}$-temperature relation is similar to that of the $38-\mathrm{keV}$ implanted samples. As the implanted dose becomes higher than $1 \times 10^{15} \mathrm{~cm}^{-2}$, the $R_{s}$ value becomes clearly more stable. Fig. 3 shows the $R_{s}$-temperature relation of the samples implanted with $\mathrm{BF}_{2}^{+}$ion 


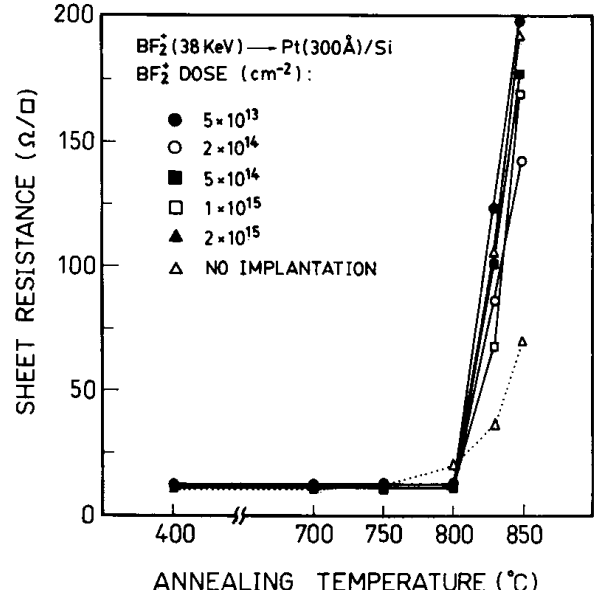

Fig. 1. $R_{s}$-temperature relation of the $\mathrm{Pt}(300 \AA) / \mathrm{Si}$ samples implanted with $\mathrm{BF}_{2}^{+}$ions at $38 \mathrm{keV}$ to various doses.

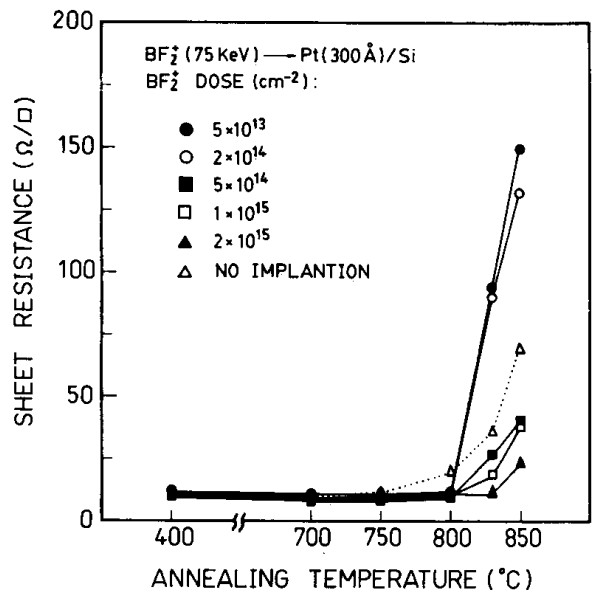

Fig. 2. $R_{s}$-temperature relation of the $\mathrm{Pt}(300 \AA) / \mathrm{Si}$ samples implanted with $\mathrm{BF}_{2}^{+}$ions at $75 \mathrm{keV}$ to various doses.

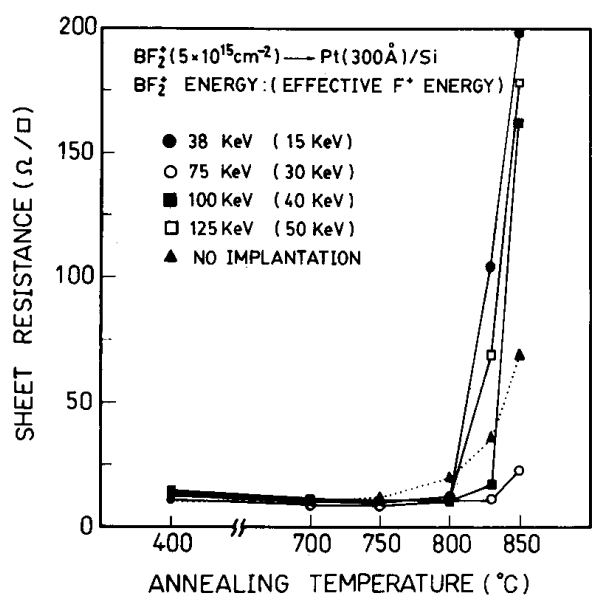

Fig. 3. $R_{\mathrm{r}}$-temperature relation of the $\operatorname{Pt}(300 \AA) / \mathrm{Si}$ samples implanted with $\mathrm{BF}_{2}^{+}$ions at $38,75,100$, and $125 \mathrm{keV}$, respectively, to a dose of $5 \times$ $10^{15} \mathrm{~cm}^{-2}$.
TABLE II

Fluorine Ion Distribution (\%) FOR THE Pt(300 $) /$ Si Structure by TRIM SiMULATION

\begin{tabular}{lrrrc}
\hline Energy $(\mathrm{keV})$ & 15 & \multicolumn{1}{c}{30} & 40 & 50 \\
\hline Backscattered & 28.54 & 24.67 & 23.65 & 21.83 \\
Within Pt film & 67.43 & 42.46 & 29.39 & 19.22 \\
$300-600 \AA$ & 4.02 & 23.57 & 22.52 & 19.22 \\
$>600 \AA$ & 0.01 & 9.30 & 24.44 & 39.73 \\
\hline
\end{tabular}

to a dose of $5 \times 10^{15} \mathrm{~cm}^{-2}$ at energies of $38,75,100$, and $125 \mathrm{keV}$ (effective $\mathrm{F}^{+}$energy of $15,30,40$, and 50 $\mathrm{keV})$, respectively. It is observed that the 75- and 100$\mathrm{keV}$ implantations give rise to the highest stability. As the implantation energy is increased to $125 \mathrm{keV}$, the hightemperature stability deteriorates.

The as-implanted fluorine distribution is simulated by the TRIM program [24]. The Pt-film thickness is assumed to be $300 \AA$. Table II lists the percentage fluorine distribution with the fluorine ion implanted at 15,30, 40, and $50 \mathrm{keV}$, respectively. The target is divided into three regions: the Pt film, the Si surface region which will be consumed during silicidation $(300-600 \AA)$, and the $\mathrm{Si}$ substrate underneath the final $\mathrm{PtSi} / \mathrm{Si}$ interface $(>600$ $\AA$ ). The thickness of the second region is calculated by multiplying the Pt-film thickness by the reacted volume ratio of $\mathrm{Pt} / \mathrm{Si}=1 / 1.32$ [1]. Because the oxygen contamination is not well controlled in the film's deposition system, the Pt film cannot be converted into PtSi completely. In this work, the final PtSi film thickness of the $\mathrm{Pt}(300$ $\AA$ ) / Si sample is about $450 \AA$ measured by ellipsometry [25] and TEM cross sections. The consumed silicon is thus about $300 \AA$ thick. With fluorine energy at $15 \mathrm{keV}$, most of the fluorine ions are confined in the Pt film. During silicide formation, this fluorine might diffuse out of the sample to the ambient and play no role on the high temperature stability. As the fluorine energy increases from 30 to $50 \mathrm{keV}$, the amount of fluorine ions that penetrate into the $\mathrm{Si}$ substrate increases. However, the maximum stable temperature of the $50-\mathrm{keV}(\mathrm{F})$ implanted samples is lower than that of the 30- and 40-keV (F) implanted samples. It is thus assumed that only the fluorine ions located near the $\mathrm{Pt} / \mathrm{Si}$ interface, especially those located within the second region, will influence the hightemperature behavior of the $\mathrm{PtSi} / \mathrm{Si}$ structure. This is quite reasonable since during the silicide formation, the fluorine atoms will be pushed ahead by the PtSi phase front to pile up at the interface.

To verify whether the optimum implantation energy is the energy at which the largest amount of fluorine ions is placed within the Si surface region to be consumed, the high-temperature stability of samples with $170 \AA$ of $P t$ was examined. Table III lists the TRIM simulated percentage fluorine distribution of the fluorine ion implanted into the $\mathrm{Pt}(170 \AA) / \mathrm{Si}$ structure at 15,20 , and $25 \mathrm{keV}$, respectively. In this case, the silicon consumption is about $170 \AA$ thick. It is observed that $20-\mathrm{keV}$ implantation places the largest amount of fluorine within the region consumed by silicidation. Thus $20 \mathrm{keV}$ should be the op- 


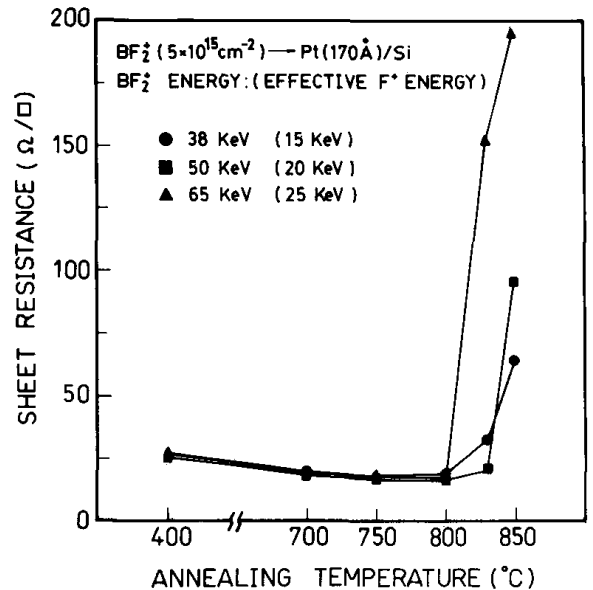

Fig. 4. $R_{s}$-temperature relation of the $\mathrm{Pt}(170 \AA) / \mathrm{Si}$ samples implanted with $\mathrm{BF}_{2}^{+}$ions at 38,50 , and $65 \mathrm{keV}$, respectively, to a dose of $5 \times 10^{15}$ $\mathrm{cm}^{-2}$.

TABLE III

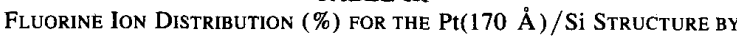
TRIM SIMULATION

\begin{tabular}{lrcc}
\hline Energy $(\mathrm{keV})$ & 15 & 20 & 25 \\
\hline Backscattered & 27.79 & 26.37 & 24.36 \\
Within Pt film & 40.20 & 26.88 & 18.18 \\
$170-340 \AA$ & 22.21 & 22.74 & 20.77 \\
$>340 \AA$ & 9.80 & 24.09 & 36.69 \\
\hline
\end{tabular}

timum energy for the $\mathrm{Pt}(170 \AA) / \mathrm{Si}$ structure. Fig. 4 shows the $R_{s}$-temperature relation of the $\operatorname{Pt}(170 \AA) / \mathrm{Si}$ samples implanted with $\mathrm{BF}_{2}^{+}$ion at 38,50 , and $65 \mathrm{keV}$ (effective $\mathrm{F}^{+}$energy of 15,20 , and $25 \mathrm{keV}$ ), respectively. This confirms the criteria of the optimum implantation energy. A similar experimental procedure was also followed for the ITS scheme. The optimum energy of fluorine ion for the PtSi $(600 \AA) / \mathrm{Si}$ structure was determined to be 50 $\mathrm{keV}$.

\section{B. $F^{+}$Implantation}

Fluorine ions alone were implanted into the $\operatorname{Pt}(300$ $\AA$ ) $/ \mathrm{Si}$ structure at the optimum energy $(40 \mathrm{keV})$ to further examine the high-temperature characteristics of the fluorinated $\mathrm{PtSi} / \mathrm{Si}$ structure. The $\operatorname{Pt}(300 \AA) / \mathrm{Si}$ structure was prepared using the same process as that employed in the $\mathrm{BF}_{2}^{+}$implantation experiment. Fluorine ions were then implanted into wafers at $40 \mathrm{keV}$ to various doses ranging from $1 \times 10^{14}$ to $5 \times 10^{15} \mathrm{~cm}^{-2}$. Following the $\mathrm{F}^{+}$implantation, all wafers were cut into smaller pieces and were annealed in $\mathrm{N}_{2}$ furnace for $60 \mathrm{~min}$ at 400,700 , $750,800,830$, and $850^{\circ} \mathrm{C}$, respectively. The unreacted Pt was selectively etched by aqua regia.

Fig. 5 shows the $R_{\mathrm{s}}$-temperature relation of the fluorine-implanted samples; the $\boldsymbol{R}_{s}$-temperature data of the unimplanted samples are also included for comparison. Since the unimplanted samples and the fluorine implanted

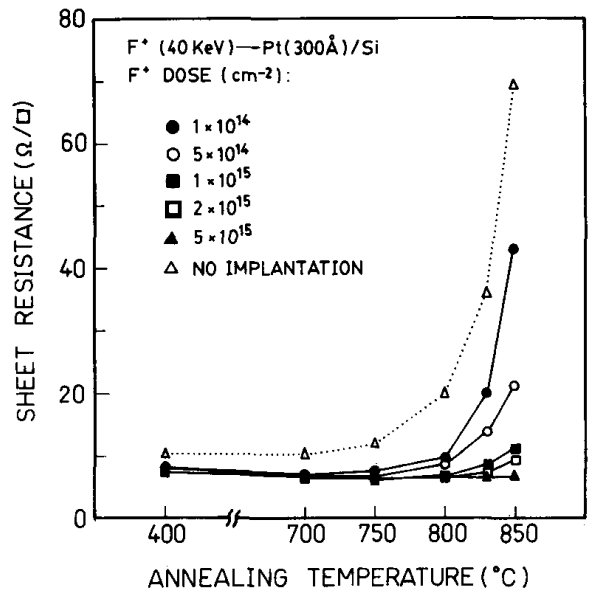

Fig. 5. $R_{s}$-temperature relation of the $\operatorname{Pt}(300 \AA) / S i$ samples with fluorine implantation at $40 \mathrm{keV}$ to various doses.

samples were not prepared at the same time and a deviation of the Pt film thickness of about $\pm 10 \%$ exists in our laboratory, sheet resistances of the unimplanted and fluorine-implanted samples at low temperature are slightly different. For the unimplanted samples, the $R_{s}$ value increases at temperatures higher than $700^{\circ} \mathrm{C}$. As the implanted fluorine dose increases from $1 \times 10^{14}$ to $5 \times 10^{15}$ $\mathrm{cm}^{-2}$, the high-temperature stability of the $R_{s}$ value of the PtSi film is successively improved. It is seen that the $R_{s}$ value remains stable up to $800^{\circ} \mathrm{C}$ as the implanted dose exceeds $1 \times 10^{15} \mathrm{~cm}^{-2}$ and up to $850^{\circ} \mathrm{C}$ as the implanted dose is $5 \times 10^{15} \mathrm{~cm}^{-2}$. Such an improvement is stable and reproducible over at least six different experimental lots.

SEM inspection was employed to further characterize the high-temperature stability of the PtSi film. Fig. 6 shows SEM micrographs of the PtSi surface and the corresponding silicon surface after removal of the silicide film of $800^{\circ} \mathrm{C}$ annealed samples without fluorine implantation and with fluorine implantation to a dose of $1 \times 10^{15}$ and $5 \times 10^{15} \mathrm{~cm}^{-2}$, respectively. It is shown that severe agglomeration occurs for the unimplanted sample, and the PtSi film becomes discontinuous. For the sample with fluorine implantation to a dose of $1 \times 10^{15} \mathrm{~cm}^{-2}$, the PtSi film is non-uniform and shows some agglomeration, and small pinholes were observed. As the implanted dose increases to $5 \times 10^{15} \mathrm{~cm}^{-2}$, the PtSi film remains smooth after $800^{\circ} \mathrm{C}$ annealing as seen in the micrograph. The morphology of the silicon surface is consistent with that of the PtSi surface. As the film agglomerates, the PtSi spikes into the silicon substrate; thus the silicon surface becomes rather rough. Fig. 7 shows SEM micrographs of the PtSi surface and the silicon surface after removal of the silicide film of $850^{\circ} \mathrm{C}$ annealed samples without fluorine implantation and with fluorine implantation to a dose of $5 \times 10^{15} \mathrm{~cm}^{-2}$. The PtSi film of the unimplanted sample has agglomerated to isolated islands which accounts for the dramatic increase of the $R_{s}$ value. For the sample 
(a)
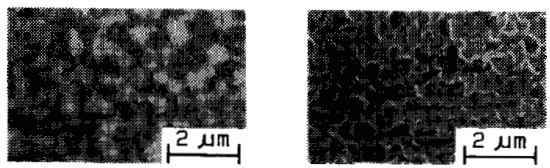

(b)
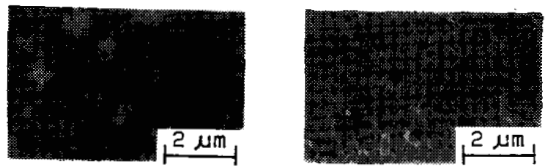

(c)
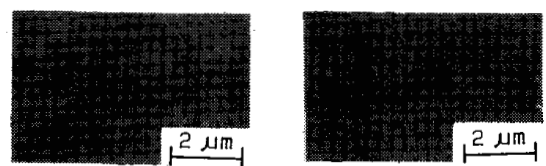

Fig. 6. SEM micrographs of the PtSi surface (left) and the Si surface after removal of the PtSi film (right) of the $800^{\circ} \mathrm{C}$ annealed samples (a) without fluorine implantation, and with fluorine implantation to a dose of (b) $1 \times$ $10^{15} \mathrm{~cm}^{-2}$ and (c) $5 \times 10^{15} \mathrm{~cm}^{-2}$.

(a)
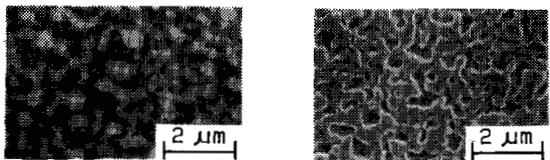

(b)
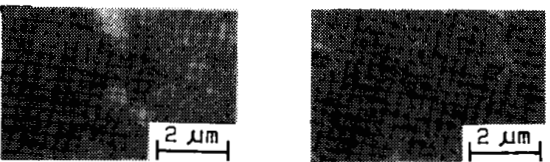

Fig. 7. SEM micrographs of the PtSi surface (left) and the Si surface after removal of the PtSi film (right) of the $850^{\circ} \mathrm{C}$ annealed samples (a) without fluorine implantation, and (b) with fluorine implantation to a dose of $5 \times$ $10^{15} \mathrm{~cm}^{-2}$

with fluorine implantation to a dose of $5 \times 10^{15} \mathrm{~cm}^{-2}$, the PtSi film becomes nonuniform but still remains continuous.

Fig. 8 shows the AES depth profiles of the samples implanted with $\mathrm{F}^{+}$ions to a dose of $1 \times 10^{14}, 1 \times 10^{15}$, and $5 \times 10^{15} \mathrm{~cm}^{-2}$, respectively, followed by an $850^{\circ} \mathrm{C}$ annealing; also shown is the profile of an unimplanted sample annealed at $400^{\circ} \mathrm{C}$. No platinum penetration into the silicon substrate is observed up to $850^{\circ} \mathrm{C}$ for the $5 \times 10^{15}$ $\mathrm{cm}^{-2}$ implanted samples. As the implantation dose decreases to $1 \times 10^{15} \mathrm{~cm}^{-2}$ or less, the PtSi film agglomerates to discontinuous islands, platinum penetrates into the silicon substrate, and the Si substrate is exposed. The RBS profile indicates similar results. These are consistent with the results of the sheet resistance measurement and the SEM inspection.

\section{Schottky-Junction Characteristics}

Because the Schottky-barrier height is very sensitive to the properties of the silicide/silicon interface [7], [8], in-

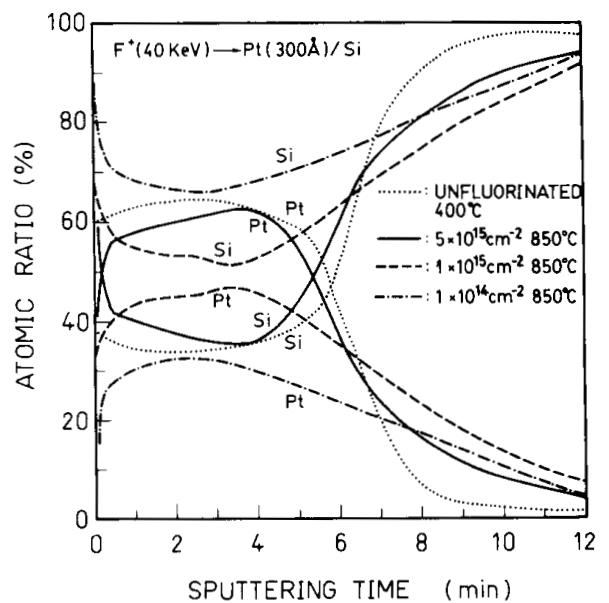

Fig. 8. AES depth profiles of the samples annealed at $850^{\circ} \mathrm{C}$ which are implanted with $\mathrm{F}^{+}$ions to a dose of $1 \times 10^{14}, 1 \times 10^{15}$, and $5 \times 10^{15}$ $\mathrm{cm}^{-2}$, respectively, and the depth profile of an unimplanted sample annealed at $400^{\circ} \mathrm{C}$

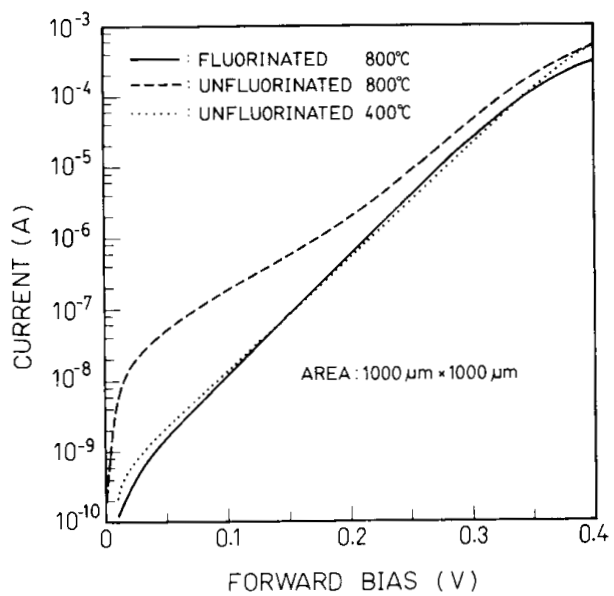

Fig. 9. Comparison of the forward $I-V$ characteristics of the Schottky junctions with fluorine implantation to a dose of $5 \times 10^{15} \mathrm{~cm}^{-2}$ and annealed at $800^{\circ} \mathrm{C}$ and the junctions without fluorine implantation and annealed at 400 and $800^{\circ} \mathrm{C}$, respectively.

vestigation on Schottky junction is used to evaluate the applicability of the fluorine implantation technique to a typical integrated circuit process for the improvement of high-temperature stability of PtSi film. The junction area was defined by etching a square window through a $4700-$ $\AA$-thick thermally grown $\mathrm{SiO}_{2}$ layer. The wafer backside was metallized by aluminum. Other processes are the same as those described in Section II-B.

Fig. 9 compares the forward $I-V$ characteristics of the $\mathrm{PtSi} / \mathrm{n}-\mathrm{Si}$ Schottky junction formed by annealing at $800^{\circ} \mathrm{C}$ with fluorine implantation to a dose of $5 \times 10^{15}$ $\mathrm{cm}^{-2}$ and the unimplanted Schottky junction annealed at 400 and $800^{\circ} \mathrm{C}$, respectively. Since the silicide/silicon interface becomes irregular at temperatures higher than $700^{\circ} \mathrm{C}$, the $800^{\circ} \mathrm{C}$ annealed, unfluorinated Schottky junc- 


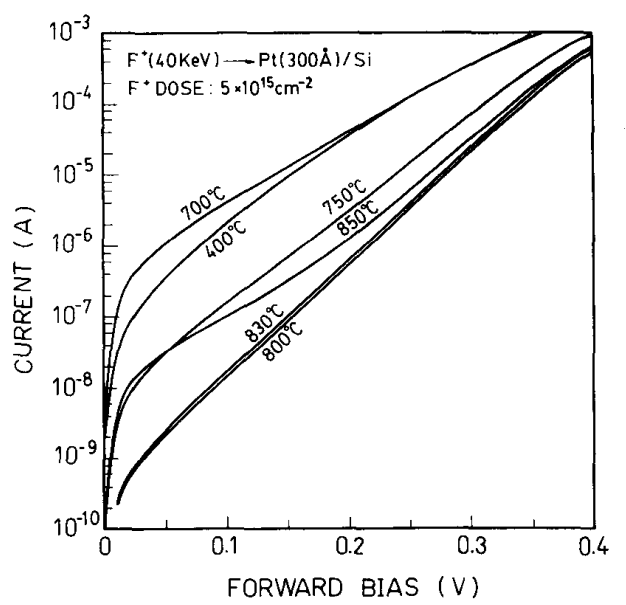

Fig. 10. Comparison of the forward $I-V$ characteristics of the Schottky junctions with fluorine implantation to a dose of $5 \times$ $10^{15} \mathrm{~cm}^{-2}$ and annealed at different temperatures.

TABLE IV

The Measured Schottky Barrier $(\phi$, $)$, Forward Ideality Factor $(\eta)$, and Reverse Current Density at $-5 \mathrm{~V}\left(J_{R}\right)$ OF THE SCHOTtKy JunCtions Without FluORINE IMPLANTATION AND With FluORINE IMPLANTATION TO A DOSE OF $5 \times 10^{15} \mathrm{~cm}^{-2}$

(X: without fluorine implantation. $\mathrm{F}$ : with fluorine implantation.)

\begin{tabular}{|c|c|c|c|c|c|c|c|}
\hline $\begin{array}{c}\text { Annealing } \\
\text { Temperature } \\
\left({ }^{\circ} \mathrm{C}\right)\end{array}$ & & 400 & 700 & 750 & 800 & 830 & 850 \\
\hline$\phi_{b n}(\mathrm{eV})$ & $\begin{array}{l}\mathrm{X} \\
\mathrm{F}\end{array}$ & $\begin{array}{r}0.866 \\
\pm 0.002 \\
0.706 \\
\pm 0.003\end{array}$ & $\begin{array}{r}0.841 \\
\pm 0.008 \\
0.677 \\
\pm 0.002\end{array}$ & $\begin{array}{r}0.812 \\
\pm 0.011 \\
0.786 \\
\pm 0.003\end{array}$ & $\begin{array}{r}0.797 \\
\pm 0.011 \\
0.864 \\
\pm 0.004\end{array}$ & $\begin{array}{r}0.801 \\
\pm 0.011 \\
0.858 \\
\pm 0.007\end{array}$ & $\begin{array}{r}* * * \\
\\
0.833 \\
\pm 0.005\end{array}$ \\
\hline$\eta$ & $\begin{array}{l}X \\
F\end{array}$ & $\begin{array}{r}1.005 \\
\pm 0.003 \\
1.376 \\
\pm 0.037\end{array}$ & $\begin{array}{r}1.054 \\
\pm 0.002 \\
1.691 \\
\pm 0.063\end{array}$ & $\begin{array}{r}1.135 \\
\pm 0.003 \\
1.230 \\
\pm 0.030\end{array}$ & $\begin{array}{r}1.193 \\
\pm 0.004 \\
1.054 \\
\pm 0.009\end{array}$ & $\begin{array}{r}1.194 \\
\pm 0.004 \\
1.066 \\
\pm 0.017\end{array}$ & $\begin{array}{r}* * * \\
1.146 \\
\pm 0.014\end{array}$ \\
\hline$J_{R}\left(\mu \mathrm{A} / \mathrm{cm}^{2}\right)$ & $\mathrm{X}$ & $\begin{array}{r}0.034 \\
\pm 0.007 \\
80.0 \\
\pm 4.5\end{array}$ & $\begin{array}{c}0.572 \\
\pm 0.112 \\
233.0 \\
\pm 29.8\end{array}$ & $\begin{array}{c}1.13 \\
\pm 0.447 \\
9.31 \\
\pm 3.57\end{array}$ & $\begin{array}{c}1.97 \\
\pm 0.814 \\
2.56 \\
\pm 0.53\end{array}$ & $\begin{array}{r}9.41 \\
\pm 7.29 \\
4.88 \\
\pm 1.76\end{array}$ & $\begin{aligned} & 46.2 \\
& \pm 23.8 \\
& 202 \\
& \pm 140\end{aligned}$ \\
\hline
\end{tabular}

tion has degraded severely. For the fluorinated Schottky junction, the interface remains stable up to $800^{\circ} \mathrm{C}$. Thus no evident difference is observed between the forward characteristic of the $800^{\circ} \mathrm{C}$ annealed, fluorinated junction and that of the $400^{\circ} \mathrm{C}$ annealed, unfluorinated junction. Table IV lists the measured Schottky-barrier height $\left(\phi_{b n}\right)$, the forward ideality factor $(\eta)$, and the reverse current density at $-5 \mathrm{~V}\left(J_{R}\right)$ of the Schottky junctions without fluorine implantation and with fluorine implantation to a dose of $5 \times 10^{15} \mathrm{~cm}^{-2}$. These values are obtained by averaging data from at least 10 arbitrarily chosen junctions. Also shown are their standard deviations. The $\phi_{b n}$ and $\eta$ values are extracted from the semi-log forward $I-V$ plot using a Richardson constant of $120\left(\mathrm{~A} / \mathrm{cm}^{2} / \mathrm{k}^{2}\right)$. The $\phi_{b n}$ of the $800^{\circ} \mathrm{C}$ annealed, fluorine implanted junction is close to that of the $400^{\circ} \mathrm{C}$ annealed, unimplanted junction. This indicates that most of the implantation damages near the silicide/silicon interface has been recovered, and the Schottky barrier is restored to its normal feature. The ideality factor $(\eta)$ of fluorine-implanted junctions is lowest after $800^{\circ} \mathrm{C}$ annealing, but it is slightly higher than that of $400^{\circ} \mathrm{C}$ annealed, unimplanted junctions. Fig. 10 compares the forward $I-V$ characteristics of the Schottky junctions with fluorine implantation to a dose of $5 \times 10^{15}$ $\mathrm{cm}^{-2}$ and annealed at different temperatures. The degradation of the 830 and $850^{\circ} \mathrm{C}$ annealed junction is attributed to the local nonuniformity of the PtSi film.

It is observed in Table IV that the $J_{R}$ of the fluorineimplanted junction is higher than that of the $400^{\circ} \mathrm{C}$ annealed, unimplanted junction. Even if the fluorine-implanted junction had been annealed at $800^{\circ} \mathrm{C}$ and the $J_{R}$ decreased to the lowest value, the $J_{R}$ would still be two orders of magnitude higher than that of the $400^{\circ} \mathrm{C}$ annealed unimplanted junction. Fig. 11 shows a TEM crosssectional view of the surface layer of a fluorine-implanted sample. It is observed that some dislocation loops and bubble-like defects reside in the silicon substrate. The high $J_{R}$ is thus attributed to these residual defects. 


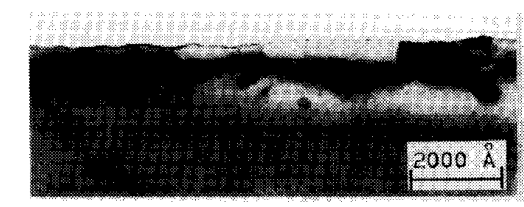

Fig. 11. TEM cross-sectional view of the $800^{\circ} \mathrm{C}$ annealed sample with fluorine implantation to a dose of $5 \times 10^{15} \mathrm{~cm}^{-2}$.

\section{Discussion}

In a previous publication [22], the authors have demonstrated by SIMS analysis that the fluorine atoms segregate at the silicide / silicon interface after thermal treatment. In this work, we try to compare the fluorine segregation behavior with different implanting dose and annealing conditions. Before samples were SIMS analyzed, the PtSi film was stripped by boiling in aqua regia to prevent the results from being interfered with by the $\mathrm{PtSi}$ film. Since residual Pt signal is still detected at the sample surface, the fluorine concentration is not quantified. Fig. 12 shows the fluorine profiles of the samples with fluorine implantation to a dose of $1 \times 10^{15}, 2 \times$ $10^{15}$, and or $5 \times 10^{15} \mathrm{~cm}^{-2}$, followed by an $800^{\circ} \mathrm{C}$ anneal. It is expected that both the total dose in the substrate and the interface concentration decrease as the implanting dose decreases. The higher the interface concentration, the better is the thermal stability, as shown by the sheet resistance data. From this observation, we find a direct relation of the thermal stability of fluorinated $\mathrm{PtSi} / \mathrm{Si}$ structure and the fluorine concentration at the interface. Another interesting phenomenon shown in the fluorine profiles is the kink at about $300 \AA$ below the interface. This could be attributed to the gettering by the residual damage [26] since heavy substrate damage was produced during the high energy implantation. A similar phenomenon was also observed by other workers [27]. This damage gettering behavior can explain why the Schottky barrier and the forward and reverse characteristics of the fluorine-implanted Schottky diode can be recovered after annealing.

The chemical state of such a high-concentration fluorine was further analyzed by the $\mathrm{X}$-ray photoelectron spectroscopy (XPS). The PtSi film was first etched by boiling in dilute aqua regia. A thin native $\mathrm{SiO}_{2}$ layer was grown during the etching process and the subsequent DI water rinsing step. The oxide layer was not removed using the fluorine-containing solution to avoid the interference of residual fluorine contamination from the solution. Instead, the sample was loaded into the chamber, and argon sputtering was employed until the Auger electron signal of oxygen decreased to the background noise level. The XPS analysis was then performed within the sputtered window. A value of $84.0 \mathrm{eV}$ for the $\mathrm{Au}\left(4 f_{7 / 2}\right)$ peak was used as reference of calibration for the XPS binding energy. The $\mathrm{F}_{1 s}$ peak at $686.2 \mathrm{eV}$ corresponds to the $\mathrm{SiF}_{2}$ or $\mathrm{SiF}_{3}$ type species [28], [29]. No atomic or molecular fluorine was detected.

The polycrystalline silicide allocated between two other

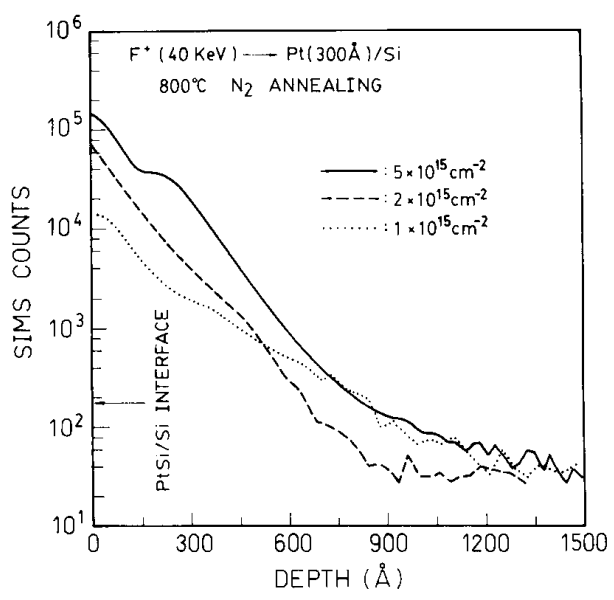

Fig. 12. Comparison of the fluorine profiles of the $800^{\circ} \mathrm{C}$ annealed samples with fluorine implantation to a dose of $1 \times 10^{15}, 2 \times 10^{15}$, and $5 \times$ $10^{15} \mathrm{~cm}^{-2}$, respectively.

phases, i.e., the silicon substrate and the ambient, is a structure which is not thermodynamically stable. The driving force for this silicide agglomeration is the reduction of total energy of the system, including that of the silicide grain boundary, the silicide/silicon interface, and the silicide-free surface [30]. The agglomeration process will continue until all the grain boundaries disappear, that is, the islanding of the silicide film, once the thermal energy can be sufficiently applied. As for the fluorine addition to the PtSi/Si system, we can expect that there will be a certain reduction of the total system energy. We are not able to distinguish whether it is a result of the lowered grain boundary energy or the interface energy. Chen et al. [31], [32] argued that the improved thermal stability of $\mathrm{NiSi}_{2}$ system is attributed to the retardation of the growth of $\mathrm{NiSi}_{2}$ grain by the presence of boron and/or fluorine atoms at the grain boundaries. We observed in our experiment that, once the implanting energy is not sufficient to have the fluorine ion reaching the silicide/silicon interface, very little effect on the thermal stability can be obtained. It is evident that the fluorine segregation at the interface, and not at the grain boundaries, plays a dominant role in the stabilization. It was also found that $\mathrm{BF}_{2}^{+}$implantation when the whole dose is confined to the silicide layer degrades the thermal stability of $\mathrm{CoSi}_{2} / \mathrm{Si}$ system [33], which further confirms our conclusion.

Because it is very difficult to explain unambiguously the stabilizing influence from the thermodynamic point of view, kinetic factors determining the transport of material, which causes the agglomeration, should also be considered. Van der hove [30] proposed a silicide dissociation model, which is associated with the solid-phaseepitaxy process [34], to explain the agglomeration phenomenon of the silicide thin film. He suggested that the agglomeration occurs through the dissociation of the silicide at the intersection of the grain boundaries and the 
interface. The dissociated silicon atoms grow epitaxially on the silicon substrate, while the metal atoms migrate through the silicide grains and react with the substrate forming new silicide at the bottom of the silicide globule. There are two kinetic limitation processes we can find in this mechanism: one is the silicon epitaxy process, the other is the metal silicide formation process. Both of them require a lot of silicon dangling bonds to which either the silicon or metal atoms can easily bond to. We found in our SIMS measurement and XPS analysis that a lot of fluorine atoms were bonded to the dangling silicon atoms at the $\mathrm{PtSi} / \mathrm{Si}$ interface because of the strong binding energy of Si-F bond of about $5.73 \mathrm{eV}$. This means that the silicon dangling bonds, which the agglomeration process demands, are no longer sufficiently supplied. The whole system will necessitate even more thermal energy to break the $\mathrm{Si}-\mathrm{F}$ bonds making the silicon dangling bonds available for the silicide film to agglomerate. This is the proposed fluorine-buffer effect and it is the fluorine-buffer model (FB model) that explains why the fluorine incorporation at the silicide/silicon interface improves the thermal stability of the system.

In fact, work has been done by other authors dealing with the thermal stability of other silicides, especially $\mathrm{TiSi}_{2}$ [35], [36]. Those results suggest several methods to prevent agglomeration. This includes lowering the process temperature and time, limiting diffusion and grain growth, or using thicker films. Other possibilities include using epitaxial single-crystal films and adding contaminants to the film which inhibit grain growth. Shenai has proposed that the thermal stability of $\mathrm{TiSi}_{2}$ at elevated temperatures is diffusion-controlled. Diffusion of titanium, silicon, dopants, defects, and film contaminants play a critical role. Thus the $\mathrm{TiSi}_{2}$ film formed on singlecrystal silicon is thermally stable at much higher post-silicide processing temperatures compared to that formed on polycrystalline silicon under similar conditions. It is believed that the proposed FB model can be applied to other silicide/silicon systems since the high-concentration fluorine can reduce the dissociation of PtSi at grain boundaries and it can also suppress the diffusion of metal and silicon atoms.

It is necessary to clarify that the fluorine addition to the silicide/silicon system can improve the thermal stability, but cannot totally inhibit the silicide film agglomeration because this system is basically not thermodynamically stable. Once a sufficient thermal energy is supplied to the system, i.e., annealing the system at higher temperature and/or for a longer period, the polycrystalline silicide film will eventually degrade. It is worth notice that the outdiffusion of fluorine from silicon substrate, the bonding of fluorine atoms to the dangling bonds of interfacial layer, the gettering of fluorine at the damage sites of the substrate, the breaking of the Si-F bonds, the dissociation of the silicide at the intersections of grain boundaries and the interface, the epitaxial growth of dissociated silicon atoms, and silicide formation at the bottom of the silicide globule are all thermally activated processes. There will be different thermal balance conditions for different thermal treatments. Fortunately, it seems that the fluorine addition to the PtSi/Si system favors the practical thermal ranges for the integrated circuit process. To make the system thermodynamically stable, epitaxial silicide may be a very promising solution [37], [38].

The FB model can also be applied to the observation of sheet resistance of the fluorinated samples as shown in Figs. 1-5. To retard the agglomeration of the silicide film, the fluorine concentration must be increased to a certain level. As the implantation dose decreases, the FB effect decreases, and the high temperature stability degrades. Besides the implantation dose, the implantation energy also plays an important role on the FB effect. The results of low energy implantation are similar to those of low dose implantation because of insufficient fluorine incorporation into the silicon substrate. Higher implantation energy introduces more fluorine atoms into the silicon substrate; however, the fluorine projected range may be too large to be far away from the silicide/silicon interface. During annealing, the formation of an efficient FB layer competes with the degradation of the silicide film. If the amount of the fluorine atoms located near the interface is not large enough, the silicide film degrades before the embedded fluorine can pile up at the interface to form the FB layer. This is consistent with the optimum implantation condition proposed in Section II-A.

\section{Conclusion}

In this work we studied the effect of fluorine incorporation on the thermal stability of the $\mathrm{PtSi} / \mathrm{Si}$ structure. Fluorine-containing species $\left(\mathrm{F}^{+}\right.$and $\left.\mathrm{BF}_{2}^{+}\right)$were implanted into the $\mathrm{Pt} / \mathrm{Si}$ and the $\mathrm{PtSi} / \mathrm{Si}$ structures followed by annealing in $\mathrm{N}_{2}$ ambient from 400 to $850^{\circ} \mathrm{C}$. During the annealing, the fluorine atoms in the $\mathrm{Si}$ substrate move toward the PtSi/Si interface and pile up there. The fluorine atoms bond to silicon atoms to form $\mathrm{SiF}_{2}-$ or $\mathrm{SiF}_{3}$-type compound. Such a highly fluorinated layer acts as a fluorine-buffer layer to change the $\mathrm{PtSi} / \mathrm{Si}$ interface energy (and/or the silicide grain boundary energy) which in turn improves the high-temperature stability of the $\mathrm{PtSi} / \mathrm{Si}$ structure. In the experimental conditions of this work, the higher the implanted fluorine dose, the better the high temperature stability. The implantation energy also plays an important role. The optimum implantation energy is the energy at which the maximum fraction of the implanted fluorine ions is placed near the $\mathrm{PtSi} / \mathrm{Si}$ interface in the Si substrate. Higher energy introduces more fluorine into the Si substrate but their distribution may be too far away from the $\mathrm{PtSi} / \mathrm{Si}$ interface so that when the $\mathrm{PtSi} / \mathrm{Si}$ structure is annealed the PtSi film may have degraded before the fluorine-buffer layer is formed. The optimum implantation energy for different $\mathrm{Pt}$ or PtSi thickness may be predicted by the TRIM simulation. Fluorineimplanted Schottky junction was fabricated to examine the applicability of the fluorinated scheme to the integrated 
circuit process. The Schottky-barrier height and the forward ideality factor of the fluorinated junctions remain comparable to those of the typical PtSi / Si Schottky junction, but the fluorine-implanted Schottky junction is able to sustain a process temperature as high as $800^{\circ} \mathrm{C}$. $\mathrm{Al}-$ though the residual defect will increase the reverse current of the fluorine-implanted Schottky junction, it will not affect the PtSi contacted $n^{+}-p$ or $p^{+}-n$ junction if the residual defect is confined within the heavily doped region. The FB model can also be employed to explain the superior high-temperature performance of the $\mathrm{CoSi}_{2}$ and $\mathrm{TiSi}_{2}$ film grown on the $\mathrm{BF}_{2}^{+}$-implanted $\mathrm{Si}$ substrate as compared to those grown on the unimplanted $\mathrm{Si}$ substrate [39], [40]. The fluorine implantation scheme will be a valuable technique in the future for the improvement of the integrated circuit reliability.

\section{ACKNOWLEDGMENT}

The authors wish to thank L. F. Wu for XPS analysis at the National Tsing-Hua University. The authors also appreciate S. Y. Wu's assistance in the implanter operation.

\section{REFERENCES}

[1] M. A. Nicolet and S. S. Lau, in VLSI Electronics: Microstructure Science, vol. 6, N. G. Einspruch and G. B. Larrabee, Eds. New York: Academic Press, 1983 , p. 329.

[2] T. Shibata, K. Hieda, M. Konaka, R. L. M. Dang, and H. Iizuka, "An optimally designed process for submicron MOSFETs," in IEDM Tech. Dig., 1981, p. 647.

[3] B. Y. Tsui and M. C. Chen, "Formation and characterization of a PtSi contacted $\mathrm{n}^{+} \mathrm{p}$ shallow junction," J. Appl. Phys., vol. 68, p. 2265,1990

[4] B. Y. Tsui, J. Y. Tsai, and M. C. Chen, "Formation of PtSi contacted $\mathrm{p}^{+}-\mathrm{n}$ shallow junctions by $\mathrm{BF}_{2}^{+}$implantation and low temperature furnace annealing,"'J. Appl. Phys., vol. 69, p. 4354, 1991.

[5] S. P. Murarka, in Silicide for VLSI Applications. New York: Aca demic Press, 1983, p. 7.

[6] A. Prabhakar, T. C. McGill, and M. A. Nicolet, "Platinum diffusion into silicon from PtSi,"' Appl. Phys. Lett., vol. 43, p. 1118,1983

[7] E. Calleja, J. Garrido, J. Piqueras, and A. Martiney, "Thermal degeneration of Mo and Pt silicon Schottky diodes," Solid-State Electron, vol. 23, p. $591,1980$.

[8] S. P. Murarka, E. Kinsbron, D. B. Fraser, J. M. Andrews, and E. J. Lloyd, "High temperature stability of PtSi formed by reaction of metal with silicon or by cosputtering," J. Appl. Phys., vol. 54, p. 6943, 1983.

[9] E. F. da Silva, Jr., Y. Nishioka, and T. P. Ma, "Radiation response of MOS capacitors containing fluorinated oxides," IEEE Trans. Nucl. Sci., vol. NS-34, p. 1190, 1987.

[10] P. J. Wright, N. Kasai, S. Inoue, and K. C. Saraswat, "Improvement in $\mathrm{SiO}_{2}$ gate dielectrics with fluorine incorporation,"' in Proc. Symp. on VLSI Technology, 1989, p. 51.

[11] Y. Nishioka, K. Ohyu, Y Ohji, N. Natsuaki, K. Mukai, and T. P. $\mathrm{Ma}$, "The effect of fluorine implantation on the interface radiation hardness of Si-gate metal-oxide-semiconductor transistors," J. Appl. Phys., vol. 66, p. 3909, 1989.

[12] "Hot-electron hardened Si-gate MOSFET utilizing F implantation," IEEE Electron Devices Lett., vol. 10, p. 141, 1989.

[13] P. J. Wright, N. Kasai, S. Inoue, and K. C. Saraswat, "Hot-electron immunity of $\mathrm{SiO}_{2}$ dielectrics with fluorine incorporation," IEEE Electron Devices Lett., vol. 10, p. 347, 1989.

[14] P. J. Wright and K. C. Saraswat, "The effect of fluorine in silicon dioxide gate dielectrics,'” IEEE Trans. Electron Devices, vol. 36, p. $879,1989$.

[15] N. Kasai, P. J. Wright, and K. C. Saraswat, "Hot-carrier-degrada- tion characteristics for fluorine-incorporated n MOSFET's," IEEE Trans. Electron Devices, vol. 37, p. 1426, 1990.

[16] Y. Ohji, Y. Nishioka, K. Yokogawa, K. Mukai, Q. Qiu, E. Arai and T. Sugano, "Effects of minute impurities $(\mathrm{H}, \mathrm{OH}, \mathrm{F})$ on $\mathrm{SiO}_{2} / \mathrm{Si}$ interfaces as investigated by nuclear resonant reaction and electron spin resonance,"' IEEE Trans. Electron Devices, vol. 37, p. 1635, 1990.

[17] F. K. Baker, J. R. Pfiester, T. C. Mele, H. H. Tseng, P. J. Tobin, J. D. Hayden, C. D. Gunderson, and L. C. Parrillo, "The influence of fluorine on threshold voltage instabilities in $\mathrm{p}^{+}$polysilicon gated p-channel MOSFET's,", in IEDM Tech. Dig., 1989, p. 443.

[18] J. M. Sung, C. Y. Lu, M. L. Chen, S. J. Hillenius, W. S. Lindenberger, L. Manchanda, T. E. Smith, and S. J. Wang, "Fluorine effect on boron diffusion of $\mathrm{p}^{+}$gate devices," in IEDM Tech. Dig., 1989, p. 447

[19] J. R. Pfiester, F. K. Baker, T. C. Mele, H. H. Tseng, P. J. Tobin, J. D. Hayden, J. W. Miller, C. D. Gunderson, and L. C. Parrillo, "The effect of boron penetration on $\mathrm{p}^{+}$polysilicon gated PMOS devices," IEEE Trans. Electron Devices, vol. 37, p. 1842, 1990

[20] J. J. Sung and C. Y. Lu, "Polysilicon-gate MOSFET's instability with fluorine incorporation," IEEE Trans. Electron Devices, vol. 37, p. 2312,1990

[21] J. Kato, "The effect of fluorine atoms in high-dose arsenic or phosphorus ion implanted silicon," J. Electrochem. Soc., vol. 137, p. $1918,1990$.

[22] J. Y. Tsai, B. Y. Tsui, and M. C. Chen, "High-temperature stability of platinum silicide associated with fluorine implantation," J. Appl. Phys., vol. 67, p. 3530, 1990.

[23] D. Gräf, M. Grundner, R. Schulz, and L. Mühlhoff, "Oxidation of HF-treated Si wafer surfaces in air," J. Appl. Phys., vol. 68, p. 5155 , 1990.

[24] J. F. Ziegler, J. P. Biersack, and U. Littmark, in The Stopping and Range of Ions in Solid. New York: Pergamon, 1985.

[25] B. Y. Tsui and M. C. Chen, "Low temperature reaction of thin film platinum ( $\leq 300 \AA$ ) with (100) silicon,"' J. Appl. Phys., vol. 68, p. $6246,1990$.

[26] M. Y. Tsai, D. S. Day, and B. G. Streetman, "Recrystallization of implanted amorphous silicon layers. II. Migration of fluorine in $\mathrm{BF}_{2}^{+}$implanted silicon," J. Appl. Phys., vol. 50, p. 188, 1979.

[27] Q. F. Wang, J. Y. Tsai, C. M. Osburn, R. Chapman, and G. E. McGuire, "Improved stability of thin $\mathrm{CoSi}_{2}$ and $\mathrm{TiSi}_{2}$ films using $\mathrm{BF}_{2}^{+}$implantation," submitted to Appl. Phys. Lett.

[28] T. J. Chuang, "Electron spectroscopy study of silicon surfaces exposed to $\mathrm{XeF}_{2}$ and the chemisorption of $\mathrm{SiF}_{4}$ on silicon," J. Appl. Phys., vol. 51, p. 2614, 1980.

[29] C. D. Stinespring and A. Freedman, "Studies of atomic and molecular fluorine reactions on silicon surfaces," Appl. Phys. Lett., vol. 48, p. 718,1986

[30] L. Van den hove, "Advanced interconnection and contact schemes based on $\mathrm{TiSi}_{2}$ and $\mathrm{CoSi}_{2}$ : Relevant issues and technological implementation," Ph.D. dissertation, Katholieke Universiteit Leuven, Leuven, Belgium, June 1988.

[31] W. J. Chen and L. J. Chen, "Interfacial reaction of nickel thin films on $\mathrm{BF}_{2}^{+}$implanted (001) Si," J. Appl. Phys., vol. 70, p. 2628, 1991.

[32] _ , "Thermal stability of $\mathrm{NiSi}_{2}$ on high dose ion-implanted (001) Si,"' J. Appl. Phys., vol. 71, p. 653, 1992.

[33] J. Y. Tsai, private communication.

[34] S. S. Lau, Z. L. Liau, and M. A. Nicolet, "Solid state epitaxy in silicide forming systems," Thin Solid Films, vol. 47, p. 313, 1977.

[35] T. P. Nolan, R. Sinclair, and R. Beyers, "Modeling of agglomeration in polycrystalline thin films: Application to $\mathrm{TiSi}_{2}$ on a silicon substrate," J. Appl. Phys., vol. 71, p. 720, 1992.

[36] K. Shenai, "Thermal stability of $\mathrm{TiSi}_{2}$ films on single crystal and polycrystalline silicon,"'J. Mater. Res., vol. 6, p. 1502, 1991.

[37] H. Ishiwara, K. Hikosaka, and S. Furukawa, "Channeling and backscattering studies of the crystalline perfection and the thermal stability of epitaxial PtSi films on Si," J. Appl. Phys., vol. 50, p. 5302, 1979.

[38] S. L. Hsia, T. Y. Ten, P. Smith, and G. E. McGuire, "'Formation of epitaxial $\mathrm{CoSi}_{2}$ films on (001) silicon using Ti-Co alloy and bimetal source materials," J. Appl. Phys., vol. 70, p. 7579, 1991.

[39] W. Lur and L. J. Chen, "Interfacial reactions of cobalt thin films on $\mathrm{BF}_{2}^{+}$ion-implanted (001) silicon," J. Appl. Phys., vol. 64, p. 3505, 1988 .

[40] W. Lur and L. J. Chen, "Interfacial reactions of titanium thin films on $\mathrm{BF}_{2}^{+}$-implanted (001) Si," J. Appl. Phys., vol. 66, p. 3604, 1989. 


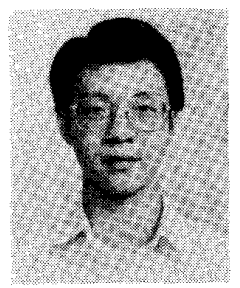

Bing-Yue Tsui (S'87) was born in Chiayi, Tajwan, in 1963 . He received the B.S., M.S., and Ph.D. degrees from the National Chiao-Tung University, Hsinchu, Taiwan, in 1985, 1987 and 1992, respectively, all in electrical engineering.

During his graduate study, he has been interested in the study of metal silicides relevant to VLSI application and electron devices physics, characterization, and reliability study. He will join ERSO, ITRI in Hsinchu, Taiwan in October, 1992 to work on submicrometer process integration.

Dr. Tsui is a member of Phi Tau Phi.

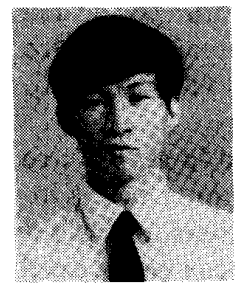

Jiunn-Yann Tsai (S'89) was born in Taichung, Taiwan, in 1965. He received the B.S. and M.S. degrees from National Chiao-Tung University, Hsinchu, Taiwan, in 1987 and 1989, respectively, both in electronic engineering.

From 1986 to 1987 , he worked on the radiation damage of e-beam metallization system to the MOS devices; from 1987 to 1989 , his work was focused on the platinum silicide and shallow junction formation. He is presently a graduate student at North Carolina State University, Raleigh, working toward the Ph.D. degree on metal silicide study relevant to VLSI application.

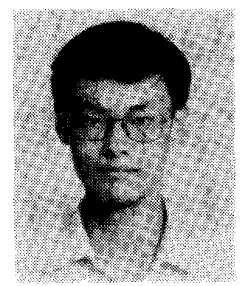

Tzong-Shien Wu was born in Taipei, Taiwan, on April 26, 1966. He received the B.S.E.E. degree from Tatung Institute of Technology, Taipei, Taiwan, in 1988 , and the M.S.E.E. degree from National Chiao-Tung University, Hsinchu, Taiwan, in 1990 .

In 1990, he joined the United Microelectronics Corporation, Process Development Division Hsinchu, Taiwan, as a process integration engineer. Since then he has been engaged in research and development of submicrometer SRAM tech-

nology.

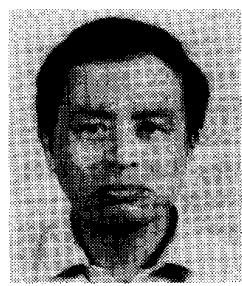

Mao-Chieh Chen was born in Tainan Hsien, Taiwan, in 1939. He received the B.S.(EE) degree from the National Taiwan University, Taipei, Taiwan, in 1961, the M.S.(EE) degree from the National Chiao-Tung University, Hsinchu, Taiwan, in 1963, and the Ph.D.(EE) degree from Carnegie-Mellon University, Pittsburgh, PA, in 1969.

In 1969, he joined the General Motors Research Labs, Warren, MI, where his work was concerned with research and development of semiconductor devices. In 1973, he was appointed professor at the Department of Material Science and Engineering, National Tsing Hua University, Hsinchu, Taiwan. In 1974, he joined the faculty of the National Chiao-Tung University as a professor in the Department of Electronics Engineering. He is presently interested in studies of CVD tungsten and CVD copper as well as metal silicides relevant to VLSI application. In addition, he has served as a Visiting Scientist to IBM T. J. Watson Research Center, Yorktown Heights, NY, (1977-1978), and the Microelectronic Center of North Carolina, Research Triangle Park (1985-1986). 